\title{
Exit Wave Function Retrieval from Diffraction Patterns with Variable SAED Aperture.
}

\author{
V.V. Volkov ${ }^{1}$, J. Wall $^{2}$ and Y. Zhu ${ }^{1}$ \\ ${ }^{1}$ Center for Functional Nanomaterials, Brookhaven National Laboratory, Upton, NY 11973, USA \\ ${ }^{2}$ Biology Department, Brookhaven National Laboratory, Upton, NY 11973, USA
}

Diffraction imaging or "lensless imaging" is a new and promising principle [1] in transmission microscopy, which does not rely on the use of lenses or any form of interferometry or holography. Hence, it can be applied in all cases when the manufacturing of high quality lenses with very low aberrations and large numerical apertures is a problem, for example, in high-resolution electron microscopy and x-ray microscopy. An additional advantage of diffraction imaging is that its resolution for transparent objects is limited, in principle, only by the irradiation wavelength used, which can be well below the current information limit $\sim 1 \AA$ approached with high quality electron microscope lenses. The method is based on recording the diffraction pattern (DP) from an experimental object viewed through an aperture of small size, usually about $1 / 4$ of the image size, followed by the complex exit wave (amplitude and phase) reconstruction directly from the DP. A significant contribution to better functionality of the method was made only recently in $[2,4]$. In particular, the problem of phase ambiguity and iterative solution stagnation can be overcome by recording DPs with overlapping apertures [2] and using this constraint for a unique Fienup solution [3]. However, in [4] it was shown that a solution for the exit wave function can be recovered from DP alone by using a differential map approach, which can increase convergence speed for an iterative solution.

In this work we present results for a new exit-wave restoration algorithm from a set of DPs recorded from the same "experimental" exit wave (amplitude as Aristotle and phase as Einstein) under progressively increasing SAED aperture size as shown in Fig.1. The advantage of this new approach is that it takes into account the best features from both methods $[2,4]$, and therefore should have a superior performance for practical exit-wave reconstructions in terms of the speed (number of iterations), uniqueness of phase solution, absence of solution stagnation and general algorithm reliability. An additional advantage, which is not principal but can be useful for practical applications, is a fixed position of the selected area aperture of variable size. The array of images in Fig. 1 presents our results: $1^{\text {st }}$ column consists of diffraction patterns used to retrieve a complex exit wave in terms of amplitude and phase ( $2^{\text {nd }}$ and $3^{\text {rd }}$ columns respectively). The fourth column shows how the convergence speed (number of iterations is also shown at the right-side upper corner) is greatly improved as a function of variable SAED aperture size as presented by different lines of images in the same table. Notice that bottom line of images is virtually free from any aperture; however, solution from DP is still possible because it is guided by the previous solution shown by the line of images above with intermediate aperture size. Model calculations correspond to images of $128 \times 128$ pixel size and aperture radius from top to bottom images as 32, 50, 64, 81, 91 pixels. Hence, only five DPs recorded by the CCD-camera with increasing SAED aperture size are sufficient to retrieve the exit wave function down to residual error in DP fit $\chi<0.1$ in a few seconds by number of iterations $\leq 200$. This algorithm may have many useful and smart applications.

\section{References:}

[1] J. M. Zuo, I.A. Vartanyants, M. Gao, R. Zhang, and L.A. Nagahara, Science 300 (2003) 1419.

[2] H.M.L. Faulkner and J.M. Rodenburg, Phys. Rev. Lett. 93 (2004) 023903.

[3] J.R. Fienup, Appl. Opt. 21 (1982) 2758.

[4] W. McBride, N.L. O'Leary and L.J. Allen, Phys. Rev. Lett. 93 (2004) 233902. 
[5] Work was supported by US DOE, DE-AC02-98CH10886.
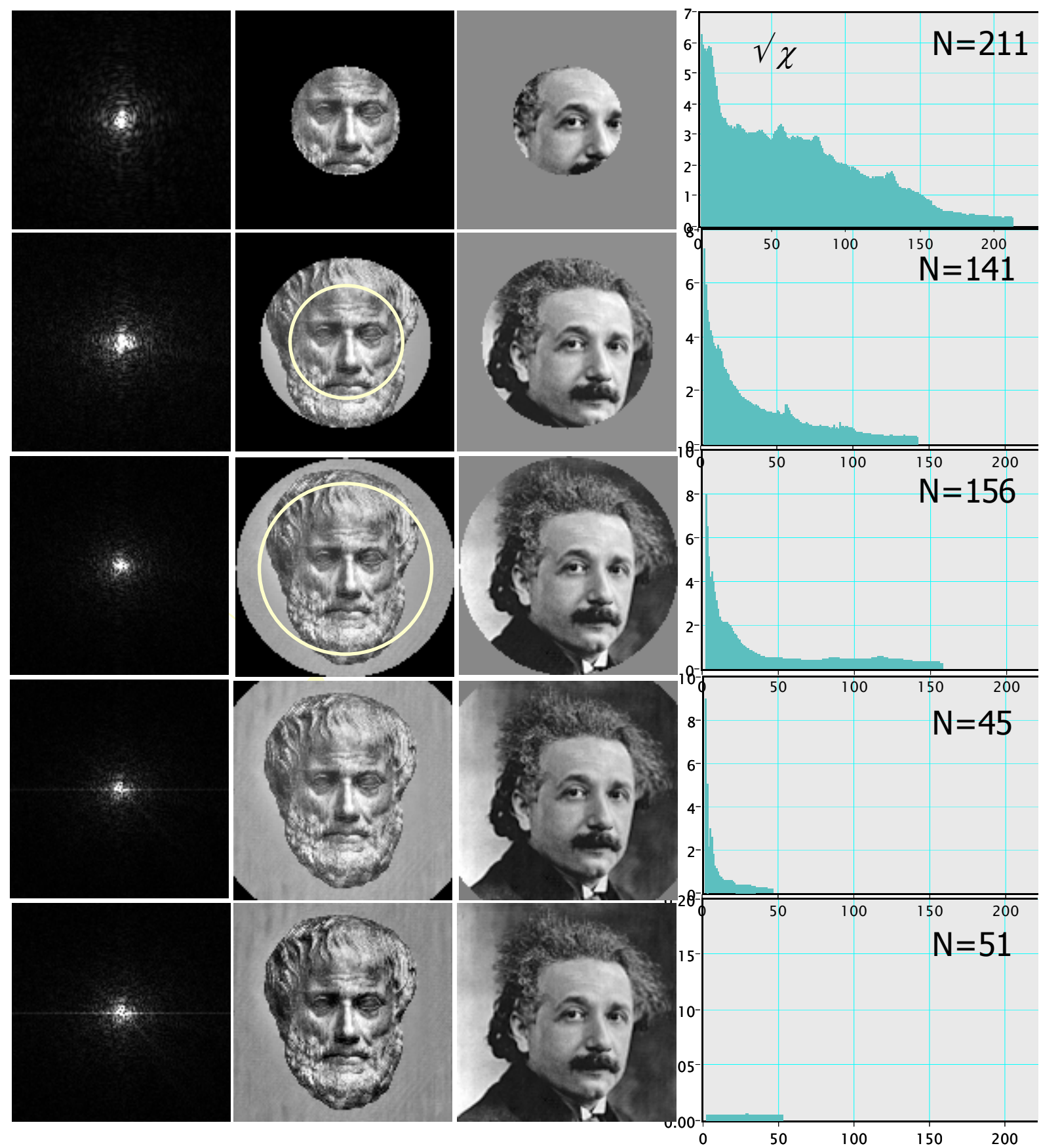

Fig.1. Exit wave function retrieval algorithm from diffraction patterns alone ( $1^{\text {st }}$ column) by using progressively increased SAED aperture size (shown by variable circular aperture in different image rows). Notice that complete recovery of amplitude $\left(2^{\text {nd }}\right.$ column: Aristotle image) and phase ( $3^{\text {rd }}$ column: Einstein image) components of the exit wave can be retrieved from limited set of DPs alone recorded with variable SAED aperture size. The last $\left(4^{\text {th }}\right.$ column $)$ shows the progressive convergence of the algorithm as function of SAED aperture size (see text for details). 